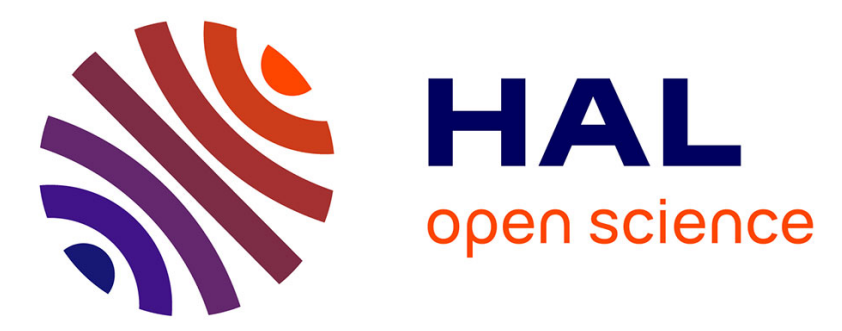

\title{
Optical Properties of Gold Nanoparticles Decorated with Furan-based Diarylethene Photochromic Molecules
}

S.V. Snegir, A.A. Khodko, D. Sysoiev, Emmanuelle Lacaze, O. Pluchery, T. Huhn

\section{- To cite this version:}

S.V. Snegir, A.A. Khodko, D. Sysoiev, Emmanuelle Lacaze, O. Pluchery, et al.. Optical Properties of Gold Nanoparticles Decorated with Furan-based Diarylethene Photochromic Molecules. Journal of Photochemistry and Photobiology A: Chemistry, 2017, 342, pp.78-84. 10.1016/j.jphotochem.2017.04.003 . hal-01504547

\section{HAL Id: hal-01504547 https: / hal.sorbonne-universite.fr/hal-01504547}

Submitted on 10 Apr 2017

HAL is a multi-disciplinary open access archive for the deposit and dissemination of scientific research documents, whether they are published or not. The documents may come from teaching and research institutions in France or abroad, or from public or private research centers.
L'archive ouverte pluridisciplinaire HAL, est destinée au dépôt et à la diffusion de documents scientifiques de niveau recherche, publiés ou non, émanant des établissements d'enseignement et de recherche français ou étrangers, des laboratoires publics ou privés. 


\title{
Optical Properties of Gold Nanoparticles Decorated
}

\section{with Furan-based Diarylethene Photochromic}

\section{Molecules}

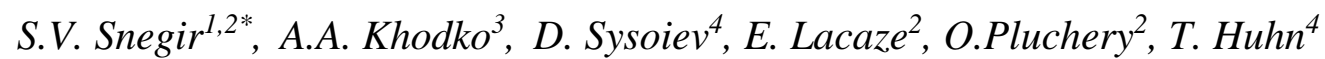

${ }^{1}$ Chuiko Institute of Surface Chemistry, National Academy of Sciences, Kyiv, Ukraine

${ }^{2}$ Institut des Nanosciences de Paris, Sorbonne Universités UPMC Univ Paris-06, CNRS-UMR 7588,

4 place Jussieu, Paris, France

${ }^{3}$ Institute of Physics, National Academy of Science, Kyiv, Ukraine

${ }^{4}$ University of Konstanz, Konstanz, Germany

*-corresponding author: ssnegir@gmail.com

Graphical Abstract

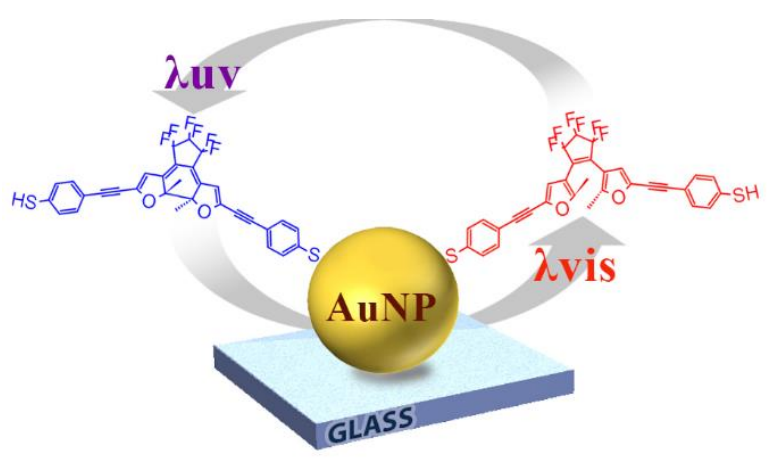

$<$ InlineImage1 $>$

\section{HIGHLIGHTS}

- OPTICAL PROPERTIES OF GOLD NANOPARTICLES DECORATED WITH FURAN-BASED DIARYLETHENE PHOTOCHROMIC

- S.V.Snegir ${ }^{1,2 *}$, A.A. Khodko ${ }^{3}$, D. Sysoiev ${ }^{4}$, E. Lacaze², O.Pluchery², T. Huhn ${ }^{4}$

- A reversible triggering of furan-based diarylethene molecules chemically attached on gold nanoparticles surface is studied

- A reversible blue shift of local surface plasmon resonance of gold nanoparticles decorated with diarylethene molecules is observed. 
- Deceleration of the ring closing kinetics of diarylethene molecules being covalently attached to gold nanoparticles is detected

\section{ABSTRACT}

The optical properties of the photochromic 1,2-bis(2-methyl-5-((4-mercaptophenyl)ethynyl)furan-3-yl)perfluorocyclopentene (YnPhT) covalently attached to gold nanoparticles (AuNP) $(\mathrm{d}=17 \pm 1 \mathrm{~nm})$ were studied in air at ambient conditions. The reversible ring-opening/closing photochromic reactions of molecules attached on AuNP surface can occur under similar light irradiation conditions as observed for free molecules in ethanolic solution. An unexpected blue shift of the local surface plasmon resonance band (LSPR) of AuNP covered by photochromic molecules was observed after UV light irradiation. A reverse red shift of LSPR was observed when the AuNP are illuminated by visible light. Moreover, increased amplitude of the LSPR was observed for AuNP covered with molecules in the closed-ring state. These observations are discussed in view of molecule - AuNP interactions. These interactions decelerate the kinetics of the ring-closing reaction of YnPhT on AuNP surfaces under UV light irradiation.

Keywords: photo-switching; ; ; ; ; ; , cyclization dynamics, furan-based diarylethene, gold nanoparticles, femtosecond pump-probe, UV/visible spectroscopy, SEM.

\section{INTRODUCTION}

There is a growing interest toward molecular switches that can be reversely triggered between two well-defined forms by external optical and/or electrical stimuli due to their potential use as ultracompact $3 \mathrm{D}$ data storage ${ }^{[1-2]}$, heavy metal sensors ${ }^{[3-5]}$, elements of nano-scaled molecular-based flexible devices ${ }^{[6-7]}$ and molecular machines ${ }^{[8-9]}$. To be implemented in a real device, photoresponsive organic molecules have to display: long-term thermal stability, good fatigue resistance, highly sensitive response to light irradiation, non-destructive readout capability and small structural changes during switching. Among the best studied photochromic molecules i.e. stilbenes, spiropyrans, azobenzenes and diarylethenes (DAE), the DAE are closest to meet the above requirements. Moreover, open $(\mathbf{O F})$ and closed form $(\mathbf{C F})$ DAE display strong variation of electric conductance ${ }^{[2]}$ when contacted by metallic electrodes, making them perfect candidates for development of optically 
driven molecular switches, an intensely investigated field during recent years ${ }^{[10-12]}$. However, further progress in design of DAE-based molecular electronics requires a detailed understanding of the photo-switching mechanism of hybrid (molecules-metallic contact) systems and the factors controlling them.

Several attempts were made to combine photochromic properties of DAE with metallic, mainly gold, flat surfaces ${ }^{[13-19]}$ or nano electrodes (nanoparticles) ${ }^{[20-27]}$ to yield hybrid materials with photochromic properties closely resembling those of DAE molecules in solution. In consequence, two main challenges were identified: The first one is the inhibition of photochromic properties (quenching) of DAE covalently attached to metallic electrodes due to strong hybridization of molecular orbitals (MO) in close vicinity to the gold surface ${ }^{[13,15,20,26,28-31]}$. This can be overcome by using a short, insulating alkyl chain as linker to decouple the photochromic core from the surface electrons i.e. reducing molecular $\pi$-electron interaction with surface atoms ${ }^{[32]}$. However, these alkyl groups are highly resistive thus diminishing applicability of the resulting hybrid system as molecular electronics element. In some studies this was overcome by adapting the linking groups to the electrode material. This efficiently separates (decouples) the MOs of the electronic system from the electrodes thus enabling at least the ring-closing reaction ${ }^{[2,18,32]}$.

The second challenge arises from coupling the plasmon resonance of the electrode with the MO of DAE. Localized surface plasmon resonsance (LSPR) is a collective excitation of the electrons of metallic nanoparticles induced by the electric field of an optical wave. For gold nanoparticles, the LSPR is excited by visible light and might be in the same energy range as the electronic transitions implied in the MO. Therefore, since LSPR and the photochromic properties of DAE occurs in the same energy range, we anticipate some mutual influences such as changes in the switching rate ${ }^{[31]}$. To address these two problems, specific structured functional groups have to be proposed and implemented in the DAE. Unfortunately, so far there is no clear understanding of what class of chemical structures might be suitable to bind photochromic molecules on metallic electrodes without degrading their optically induced reactions. Different linking groups were monitored for this purpose 
and in addition to ring-closing, the reverse ring-opening even with some enhancement of photochromic reaction on AuNP was observed in some studies. ${ }^{[24-25,27,31]}$

In one previous study we focused on the synthesis of new sulphur-free photochromic molecules based on the bisfuryl-perfluorocyclopentene core connected to different anchoring groups via $\pi$-extended wires. ${ }^{[33]}$ The influence of the anchoring group on the electrical conductance through a single-molecule junction and the alignment of the MOs to the Fermi energy level of gold electrodes were systematically studied in a gold-break junction setup. ${ }^{[34-35]}$ From this data it was concluded that for rather short molecules the nature of the anchoring group and side chain (i.e. $\pi$-extended wires) is dominating the level alignment with respect to the Fermi-level of the electrode material. Moreover, we could also show that DAE with difurylethene-based photochromic core and suitable anchoring groups switch from the $\mathrm{OF}$ to the $\mathrm{CF}$ within the picosecond ${ }^{[36]}$ or even sub-picosecond ${ }^{[37]}$ timeregime. Using these findings, flexible molecular devices based on the YnPhT DAE were designed and showed reversible conductance switching (Fig. 1) ${ }^{[10,38]}$. Apparently, YnPhT molecules sandwiched between a macroscopic non-symmetric $\mathrm{Au}$-reduced graphene-oxide electrode pair preserved their optically induced reversible switching behaviour. However, in nano-scaled or even uni-molecular electronic devices the drastically limited number of photochromic molecules has to be directly contacted by the nano electrodes which results in different electronic and photophysical properties compared to the meso or even macro scale ${ }^{[39]}$.

In the present study we use AuNP to model the surface of gold nano-electrodes and studied optical properties of $\mathrm{YnPhT}$ photochromic molecules covalently attached to them. Our aim is to monitor the light induced switching of an assembly of DAE on a nano scaled model of a gold electrode and to compare it with the reversible switching of free $\mathrm{YnPhT}$ in solution. Furthermore we want to identify the correlations between collective switching of the ensemble and the processes undergone by a single YnPhT molecule between two gold nano-electrodes in molecular break junction setup. ${ }^{\text {[35] }}$ As for the UV-induced ring-closing reaction we show that the molecule degradation is attenuated on 
gold nanoparticles. This will provide some advice to develop different approaches that drive or facilitate the electro-optical properties of molecules and devices on their basis at ambient conditions.

\section{EXPERIMENTAL SECTION}

Synthesis and purification of the acetyl-protected $\mathrm{YnPhT}$ were performed according to ref ${ }^{[35]}$. In all experiments conducted in the presence of AuNP the acetyl protecting group was removed prior to use by addition of a drop of ammonia to the solution of YnPhT to liberate the free SH form of YnPhT.

The irradiation of YnPhT in ethanolic solution by UV light $(\lambda=250-370 \mathrm{~nm})$ leads to a $6 \pi$ electrocyclization with concomitant formation of the CF (Fig.1). During irradiation the color of YnPhT solution gradually changes from transparent to violet. Consequently, the photoinduced cyclization reaction can be monitored by pump-probe spectroscopy by probing the corresponding $\mathbf{C F}$ absorption band in the visible range (discussed below).

Synthesis of gold nanoparticles was conducted according to the Turkevich protocol ${ }^{[40-43]}$. An aqueous solution of $\mathrm{HAuCl}_{4}\left(2.5 \times 10^{-4}\right.$ mol. $\left.\mathrm{L}^{-1}\right)$ was heated to the boiling point in an Erlenmeyer flask $(20 \mathrm{~mL})$. Then an aqueous sodium citrate solution $\left(1 \mathrm{~mL}, 1.7 \times 10^{-2} \mathrm{~mol} . \mathrm{L}^{-1}\right)$ was added with vigorous magnetic stirring. The colour of the solution quickly changed from transparent to light-violet ( $2 \mathrm{~min}$ ) and finally reached a stable red colour $5 \mathrm{~min}$ after citrate addition. This aqueous solution of stabilized gold nanoparticles exhibits a peak of absorbance at $\lambda_{\max }=520 \mathrm{~nm}$ (Fig.2a) in the absorption spectra. This peak corresponds to the LSPR band. An analysis of the scanning electron microscopy (SEM) images (inset of Fig. 2a) confirms that AuNP have spherical shape with average diameter of about 17 $\pm 1 \mathrm{~nm}$. The resultant solution was stored at $4^{\circ} \mathrm{C}$ to avoid nanoparticle aggregation. All chemicals were purchased from Aldrich and used as received. Glass slides $\left(10 \times 15 \mathrm{~mm}^{2}\right)$ were cut from commercially available cover slips (SCHOTT). The flatness of the surface was controlled with atomic force microscopy (AFM) measurements and the average roughness did not exceed $3 \mathrm{~nm}$ peak to peak. These slides were carefully cleaned several times in pure ethanol and dried in a flow of dry nitrogen. 
Finally, they were immersed in a methanol solution of (3-aminopropyl)-triethoxysilane (APTES) (1\%). After $3 \mathrm{~h}$ the slides were sonicated three times in fresh methanol to remove all physisorbed APTES. The pre-coated glass slides were subsequently immersed in an aqueous solution of the $\mathrm{AuNP}^{[44]}$. The immersing time used in the current experiment was 20 minutes. These slides covered by AuNP were finally rinsed several times with pure water $(18 \mathrm{M} \Omega)$ and dried in a stream of dry nitrogen. SEM reveals that AuNP are randomly dispersed on the surface with coverage of about 100 AuNP per $\mu \mathrm{m}^{2}$ (Inset, Fig. 2b). The colour of the glass became light pink due to the plasmon absorption of the AuNP under ambient light. The optical spectrum obtained in transmission, exhibits the LSPR at a wavelength of $\lambda=521 \pm 1 \mathrm{~nm}$ (Fig. 2b). The surface coverage of AuNP was controlled by the immersion-time of glass slides in the colloidal water solution of AuNP. Immersion time was chosen to obtain a coating-density in which LSPR of neighboring gold AuNP do not interfere. Therefore no additional light absorption above $530 \mathrm{~nm}$ was observed (Fig. 2b.)

Functionalization of the AuNP with the YnPhT molecules was achieved by immersion of the cover slides in an ethanolic solution of deprotected $\mathrm{YnPhT}\left(1.2 \times 10^{-4} \mathrm{~mol} . \mathrm{L}^{-1}\right)$ for $12 \mathrm{~h}$. Afterwards the cover slides were cleaned with copious amounts of fresh ethanol and dried in a stream of dry nitrogen.

The switching of YnPhT was triggered by UV or visible irradiation. A Pen-Ray® lamp (UVP, USA) with $\lambda_{\max }=254 \mathrm{~nm}\left(0.75 \mu \mathrm{W} \cdot \mathrm{cm}^{-2}\right)$ was used to induce ring closing reaction while a Schott $\mathrm{KL}$ 1500 LCD lamp (P = $150 \mathrm{~W}, \mathrm{~T}=3300 \mathrm{~K})$ was used for reverse reaction. The AFM characterizations of AuNP attachment was performed by a Bruker Multimode apparatus operating in the PeakForce ${ }^{\circledR}$ mode at ambient atmospheric conditions. UV-visible spectra were recorded using a CARY 5000 spectrometer in transmission geometry at normal incidence within the wavelength range $\lambda=400-$ $700 \mathrm{~nm}$. For measuring the modulation of the absorbance spectra when the molecule is switched, the stability of the sample holder is critical. Therefore the UV or visible irradiations were carried out directly in the spectrometer compartment without moving of the sample. The UV and visible light sources were systematically placed at $2 \mathrm{~cm}$ distance from the samples. 
SEM of glass surface covered by AuNP was performed using Zeiss Ultra55. TEM of AuNP deposited from water colloidal solution on a carbon surface was performed using Zeiss Libra120.

The formation of $\mathbf{C F}$ on the picosecond time-scale was investigated by femtosecond transient absorption spectroscopy. For these experiments, YnPhT with concentration of $\mathrm{c}=5 \times 10^{-5} \mathrm{~mol} . \mathrm{L}^{-1}$ in ethanol was used. To avoid accumulation of CF molecules, the solution was pumped through a quartz flow-cell with $2 \mathrm{~mm}$ optical path and a buffer volume by a peristaltic pump with $2 \mathrm{ml} / \mathrm{s}$ flow rate. We irradiated the solution in the buffer volume by broad visible radiation (Schott KL 1500 LCD, P = 150 W), which caused the reverse ring-opening reaction. This approach allowed refreshing of the solution in the cell with $\mathbf{O F}$ molecules by inducing $\mathbf{C F} \rightarrow \mathbf{O F}$ transformation. As for the optical setup, a Ti:Sapphire laser (Coherent, USA) was used to produce femtosecond pulses $(E=0.5 \mathrm{~mJ}, \mathrm{t}=150 \mathrm{fs}$, $\lambda=800 \mathrm{~nm}, v=1 \mathrm{kHz}$ ). This beam was split in two: the pump beam initiated the ring closing reaction, while the probe beam tracked changes of the optical density on the femtosecond timescale. The third harmonic generation was used to generate laser pulses with following parameters $\mathrm{E}=0.4 \mu \mathrm{J}, \mathrm{t}=150$ fs, $\lambda=266 \mathrm{~nm}, v=250 \mathrm{~Hz}$. As probe radiation, the supercontinuum $(\mathrm{SC}, \lambda=540-600 \mathrm{~nm})$, generated in an $\mathrm{Al}_{2} \mathrm{O}_{3}$ crystal by femtosecond pulses at $800 \mathrm{~nm}$, was used. The $\mathrm{SC}$ beam was split into probe and reference beams before entering the sample. Both beams were recorded by a multichannel spectrograph (Imaging Spectrograph SP-2500i, Acton, USA). In order to determine the time delay, the approximate zero position was obtained from mixing fundamental $\lambda=800 \mathrm{~nm}$ and $\mathrm{SC}$ in the nonlinear BBO crystal. In our data and figures the zero point position was chosen as the starting point of absorption changes. To correct SC fluctuations, the transient absorbance spectra were obtained by comparing the probe and the reference spectra for different time delays, and then, the group-velocity dispersion of SC pulse was corrected numerically at all obtained transient traces.

\section{RESULTS AND DISCUSSIONS}

Femtosecond pump-probe spectroscopy studies of ring closing reaction of $\mathrm{YnPhT}$ molecules in ethanol solution revealed an uptake of the optical density at $\lambda=540-610 \mathrm{~nm}$ when the delay between pump and probe pulses is increased stepwise up to 0.8 ps (Fig. 3). Above 0.8 ps, we detected 
only slight optical density fluctuation. No progressive spectral shifts during the first hundreds of femtoseconds after pump pulse were detected. From the normalized time profile (Fig. 3, inset) we determined the upper value of the rise-time as the characteristic of the gradual growth of optical density during the ring-closing reaction of $\mathrm{YnPhT}$ molecules to be equal to $420 \mathrm{fs}$. This value is smaller than that of thiophene based DAE ${ }^{[45-46]}$, revealing a faster ring-closing reaction of furanbased DAE functionalized by 4-mercaptophenyl-ethynyl side groups. From these results of the pump probe experiment we expect that furans grafted onto AuNP will also show a faster cyclization dynamics compared with thiophenes.

The glass slides covered with AuNP decorated by YnPhT molecules were characterized by UV-visible spectroscopy. When YnPhT molecules are covalently bound in $\mathbf{O F}$, the optical spectrum undergoes a slight modification. i.e the LSPR changes from $521 \mathrm{~nm}$ to $525 \mathrm{~nm}$ and the intensity increases by about $6 \%$ compared to bare AuNP on glass (Fig. 4a). This $6 \mathrm{~nm}$ wavelength shift accompanied with a slight increase of the absorbance is typical when a molecular layer is formed on gold nanoparticles. The small change of the refractive index in the close vicinity of the nanoparticle is modeled with the Mie theory and is largely documented ${ }^{[47-49]}$. In our case this plasmon shift is a proof of the attachment of molecules in $\mathbf{O F}$ and was discussed previously ${ }^{[50]}$. The concomitant increase of the absorbance is typical for hybrid systems where molecules are grafted onto nanoparticles and do not induce any electronic effect or any charge transfer. We deduce from recent observations done with STM on Au(111) surface covered by YnPhT molecules ${ }^{[50]}$, that the rather low increase in signal intensity is in agreement with a relatively low coverage of the $\mathrm{YnPhT}$ on the AuNP surface.

Upon irradiation of the glass slide by UV light for 6 minutes an increase of light absorbance by $22 \%$ of the overall absorbance was measured (Fig. $4 \mathrm{a}$. dashed curve). Irradiation of the sample by visible light for the same amount of time as before led to a decrease in light absorption of the glass-AuNP-YnPhT system (Fig. 4a). This behaviour is similar to the photochromic properties of 
the free YnPhT molecules in ethanolic solution (Fig.1), i.e. UV light leads to ring-closing reaction of molecules (colored solution) and visible light induces reversible ring-opening reaction (solution bleaching). Therefore, the observed changes of the light absorbance in Fig.4a can be attributed to reversible switching of YnPhT molecules covalently bound to AuNP surface, since UV/visible irradiation is known to induce no modification of the intensity of the LSPR of bare AuNP ${ }^{[51-53]}$. To demonstrate reversible switching we performed several $\mathbf{O F} \rightarrow \mathbf{C F} \rightarrow \mathbf{O F}$ cycles (Fig. 4b). These confirmed that the material interacts efficiently with UV as well as visible light and possesses pronounced photochromic properties. However, some experimental effects were observed and will be discussed in the following paragraphs.

The $\mathbf{C F} \rightarrow \mathbf{O F}$ transition under visible light irradiation (Fig.4) leads to a red shift of LSPR and lowering of the overall light absorbance. A complete recovering of the absorption spectra during $\mathbf{O F} \rightarrow \mathbf{C F} \rightarrow \mathbf{O F}$ cycles was not observed (Fig.4a). Such effect is not related to some specific properties of AuNP-YnPhT hybrid material. It represents intrinsic properties of all DAE photochromic molecules including YnPhT i.e. $\mathbf{O F} \rightarrow \mathbf{C F}$ transition under UV irradiation has a higher quantum yield than the reverse $\mathbf{C F} \rightarrow \mathbf{O F}$ under visible light irradiation ${ }^{[2]}$.

$<$ InlineShape1>

Interestingly, an increase in the light absorbance upon $\mathbf{O F} \rightarrow \mathbf{C F}$ transition is not accompanied by a red-shift of the LSPR as it might be expected when dielectric constant is increasing according to 
Mie theory ${ }^{[39]}$. Instead the $\lambda_{\max }$ of LSPR is blue-shifted by $5 \mathrm{~nm}$ upon UV irradiation of initially attached molecules to initiate the $\mathbf{O F} \rightarrow \mathbf{C F}$ transition (red solid curve $\rightarrow$ blue dashed curve; Fig.4a). This was observed systematically for different samples each time when they were subjected to UV light. This blue-shift might have two origins. One might be a simple additive effect of the absorption spectra of bare AuNP, i.e. the LSPR at $521 \mathrm{~nm}$ (Fig. 4a, black curve) with the intrinsic absorption of CF molecules ( $\lambda_{\max }$ at $554 \mathrm{~nm}$; Fig. 1, dashed curve). However, since $\mathbf{C F}$ in solution exhibits a broad absorption band (Fig. 1), the additive effect would favor an overall red shift of the LSPR as recently shown by Nishi [54]. Seemingly the observed blue shift in our experiments is not simply based on variation of the refractive index during $\mathbf{O F} \rightarrow \mathbf{C F}$ switching. In agreement with theoretical calculations for other DAE-NP hybrids which are predicting an electronic interaction ${ }^{[55-56]}$, it seems more plausible that a specific interaction between AuNP and molecular-orbitals of the closed form of YnPhT is present in our system, which enhances the impact onto the optical properties of $\mathrm{YnPhT}$ molecules. To confirm this, we subtracted the spectrum of unmodified AuNP on glass from the ones corresponding to YnPhT modified AuNP after alternating UV-/visible-irradiation cycles (Fig. 5).

The resulting spectra describe the interaction of AuNP and YnPhT (in optical meaning) dynamically as a function of YnPhT ring-opening/-closing reaction. The spectra are characterized by a prominent absorption band centered at $\lambda_{\max }=547 \mathrm{~nm}$ (Fig. 5 , red curve). Upon irradiation with UV to initiate $\mathbf{O F} \rightarrow \mathbf{C F}$ transition the overall light absorbance in the visible region increases strongly with a concomitant blue-shift of the absorption band by $15 \mathrm{~nm}$ to $\lambda_{\max }=532 \mathrm{~nm}$ (Fig. 5, blue dotted curve). This specific absorption band in the region of $540 \mathrm{~nm}$ was observed systematically in all curves of Fig. 5. Initially, we suspected a contamination of our OF-solution by a small amount of $\mathbf{C F}$ molecules during AuNP modification. However, under repetitive UV-/visible- irradiation the intensity of this band remains unchanged relative to its background. This revealed independence of this absorption band from the process of ring closing/opening of YnPhT molecules. Moreover, the width of this band is about $70 \mathrm{~nm}$ whereas the $\mathbf{C F}$ of YnPhT has a much broader (410-680 nm) 
intrinsic absorption band (Fig. 1). Thus, we cannot attribute this band to the one observed for $\mathbf{C F}$ of molecules attached to AuNP. As mentioned above, upon UV-irradiation the position of the absorption band is slightly blue shifted, whereas it is back-shifted to the red region when irradiated by visible light. Based on these findings, we hypothesize that this band results from an interaction of specific molecular orbitals of terminal 4-mercaptophenyl-ethynyl linker groups with AuNP. However, this hypothesis requires additional clarification by theoretical calculations of MOs for YnPhT linked to AuNP.

Nevertheless, the reversible increase of absorbance during alternating irradiation with either UV or visible light clearly indicates a switching process (Fig. 5). Seemingly, the photochromic properties of the hexafluorocyclopentene core of $\mathrm{YnPhT}$ on the surface of AuNP are not quenched. We attribute this to the linker groups efficiently decoupling the photochromic core from electronic states of the AuNP. These groups act as a barrier for surface electrons aiming to occupy lowest unoccupied MO during YnPhT switching. Thus, it shows that photo-excited MO are not mixed with Au surface states like in the case of thiophene-base linkers which led consequently to partial loss of photochromic properties ${ }^{[13,21]}$. At the same time our linker provides sufficient $\pi$-conjugation in electronic transport measurements, as evidenced by recent molecular-break junction experiments ${ }^{[35]}$.

To investigate the influence of the AuNP on the switching kinetics of surface bound YnPhT we compared the optical response of the AuNP-YnPhT system toward irradiation with that of YnPhT in solution. We increased the duration of UV irradiation of the glass-AuNP-YnPhT sample stepwise and measured the value of light absorbance at $554 \mathrm{~nm}$ after each irradiation cycle (Fig.6a). A sample of YnPhT in ethanol solution was treated exactly the same way (Fig.6b). Both samples were irradiated in two series with increments of 10 and 30s UV irradiation, respectively.

In experiments with the glass-bound AuNP-YnPhT system with $10 \mathrm{sec}$ increments the intensity of light absorption at $554 \mathrm{~nm}$ is linear increasing (Fig.6a). Using longer increments of $\Delta \mathrm{t}=$ 30s lead to non-linear and more progressive increasing of light absorbance during first two cycles followed by deceleration over remaining cycles (Fig.6a). 

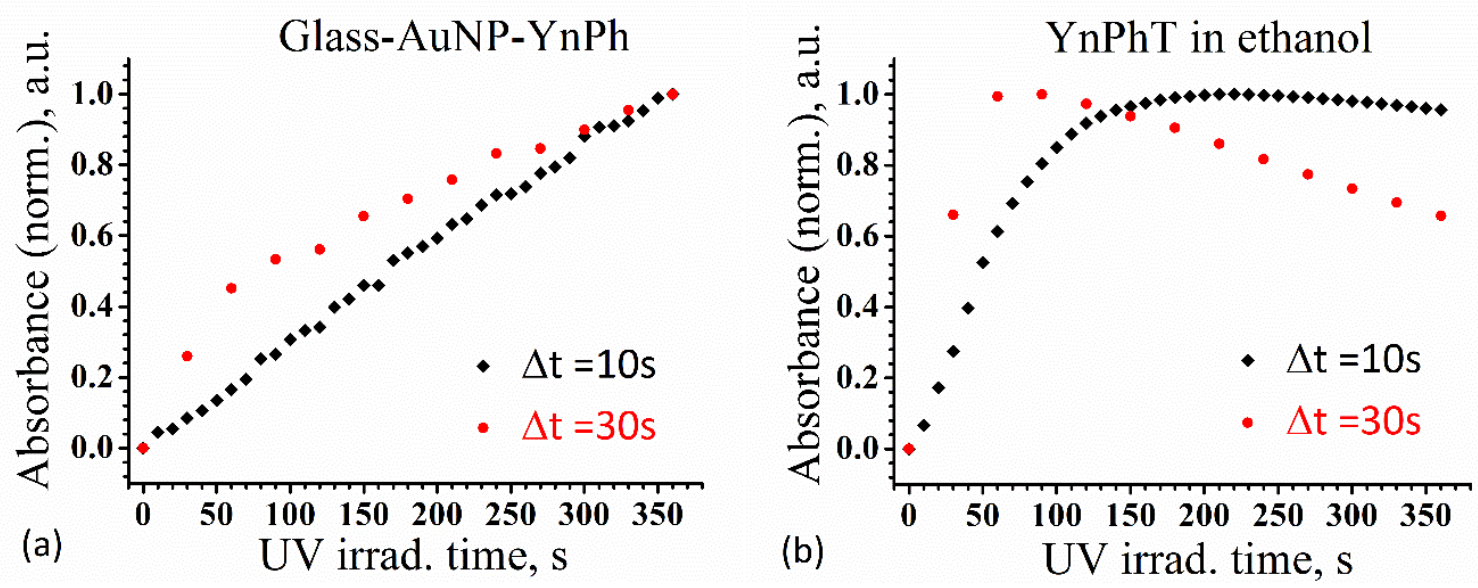

Figure.6 Evolution of absorbance change of (a) glass-NPs-YnPhT sample in air and (b) of YnPhT molecules in ethanol solution under UV irradiation $(\lambda=254 \mathrm{~nm})$ in increments of either 10s (black diamonds) or 30s (red dots) illumination time. Absorbance is measured at $554 \mathrm{~nm}$ after each irradiation cycle.

Repeating the experiment with unbound YnPhT molecules in solution (Fig.6b) resulted in an initially fast absorbance increase which reached quickly saturation. The process occurs more rapidly in the experiment with 30s. increment whereas the experiment with shorter irradiation intervals of $10 \mathrm{sec}$ took longer to reach saturation (Fig. 6b). Independent of the irradiation time, the absorbance increase is much faster in solution than in the AuNP-YnPhT hybrid system (Fig.6b). Saturation is reached in solution either after 60 s (30s intervals) or 150s (10s intervals) of accumulated UV irradiation whereas the AuNP-YnPhT system does not achieve saturation even after 350s of accumulated UV irradiation. Longer UV irradiation of YnPhT solution leads to a slow decrease of light absorbance mainly by decomposition of $\mathrm{YnPhT}^{[57]}$.

The kinetics of ring-closing reaction of YnPhT molecules chemically attached to AuNP surface is thus different from the one of free $\mathrm{YnPhT}$ in solution. During continuous UV irradiation of ethanolic solution all $\mathrm{YnPhT}$ molecules are transformed to $\mathbf{C F}$ much more rapidly than the one attached to AuNP surface. YnPhT molecules attached to AuNP apparently show a slower ring closing 
reaction. This observation is consistent with our recent UV/visible light switching experiments for YnPhT anchored to a flat gold mono crystal ${ }^{[10]}$. These experiments revealed higher stability of DAE on a metallic surface under mechanical and temperature stresses albeit with reduced switching kinetics. This effect can be explained by modification of the HOMO-LUMO gap which is responsible for the ring-closing reaction of $\mathrm{YnPhT}$ on a gold surface. The effect of HOMO-LUMO gap modification was experimentally proven in break-junction measurements ${ }^{[35]}$ and predicted theoretically for other DAE attached to small gold cluster ${ }^{[56]}$. Consequently this leads to different quantum yields for the $\mathbf{O F} \rightarrow \mathbf{C F}$ transformation of free $\mathrm{YnPhT}$ in solution and the ones connected to AuNP.

Unexpectedly, our experiments show a slightly different kinetic of ring-closing reaction when different irradiation increments were used. Samples irradiated with longer intervals of UV irradiation show a stronger increase of absorbance over time than those samples which were irradiated using 10 sec intervals. Whereas in the AuNP-YnPhT hybrid sample both curves merge after a total of $\sim 300$ $\mathrm{sec}$, the situation in solution is different. Here samples irradiated in $30 \mathrm{sec}$ intervals reach saturation faster and start decomposition while samples irradiated by shorter intervals of UV light lag behind. We do not know yet if the solvent is involved in this process. At present this effect remains largely unclear and demands further investigations.

\section{CONCLUSIONS}

YnPhT photochromic molecules with 4-mercaptophenyl-ethynyl linker-groups display subpicosecond cyclization reaction in solution. Pump-probe experiments revealed a faster ring closing process compared to thiophene-based analogues. Grafting AuNP $(17 \pm 1 \mathrm{~nm})$ on APTES modified glass substrates allowed the functionalization of AuNP with YnPhT molecules by standard thiol-Au bond forming technique. The thus generated hybrid system was characterized by UV spectroscopy during alternating UV/vis-irradiation cycles. The interaction of YnPhT with the gold surface does not lead to complete quenching of photochromic properties; instead it induces a moderate deceleration of the ring closing kinetics. It is expected that the optical properties of created hybrid material may 
improve if attachment geometry of molecules on AuNP surface can be controlled ${ }^{[55-56]}$. However this requires strong theoretical calculation performed in combination of molecular dynamics, DFT and TD-DFT methods.

We explain this by the presence of the 4-mercaptophenyl-ethynyl linker-group which attaches the YnPhT to the AuNP. Apparently it decouples the MO of the photochromic core from electronic states of the Au-NP thus suppressing quenching effects. These renders YnPhT molecules good candidates for the construction of nano sized organo-metallic hybrid switches with optically controlled properties.

\section{ACKNOWLEDGMENTS}

The work was supported by the DNIPRO program (2015-2016) established by Ministry of Education and Science of Ukraine and Ministry of foreign affairs of France; the Deutsche Forschungsgemeinschaft through SFB767; the FP7 Marie Curie ILSES project No 612620.

\section{REFERENCES}

[1] K. Ogawa, Appl. Sci. 2014, 4, 1-18.

[2] M. Irie, T. Fukaminato, K. Matsuda, S. Kobatake, Chem. Rev. 2014, 114, 12174-12277.

[3] H. Jia, S. Pu, C. Fan, G. Liu, C. Zheng, Dyes Pigm. 2015, 121, 211-220.

[4] D. Xue, C. Zheng, C. Fan, G. Liu, S. Pu, J. Photochem. Photobiol., A 2015, 303, 59-66.

[5] C. Zhang, S. Pu, Z. Sun, C. Fan, G. Liu, J. Phys. Chem. B 2015, 119, 4673-4682.

[6] Y. Ishibashi, M. Mukaida, M. Falkenstroem, H. Miyasaka, S. Kobatake, M. Irie, Phys. Chem. Chem. Phys. 2009, 11, 2640-2648.

[7] M. El Gemayel, K. Borjesson, M. Herder, D. T. Duong, J. A. Hutchison, C. Ruzie, G. Schweicher, A. Salleo, Y. Geerts, S. Hecht, E. Orgiu, P. Samori, Nat. Commun. 2015, 6, 6330.

[8] P. Naumov, S. Chizhik, M. K. Panda, N. K. Nath, E. Boldyreva, Chem. Rev. 2015, 115, 1244012490.

[9] Molecular Switches, 2nd ed., Vol. 1 (Eds.: B. L. Feringa, W. R. Browne), Wiley-VCH, Weinheim, 2011. 
[10] D. Kim, H. Jeong, H. Lee, W.-T. Hwang, J. Wolf, E. Scheer, T. Huhn, H. Jeong, T. Lee, Adv. Mat. 2014, 26, 3968-3973.

[11] T. Sendler, K. Luka-Guth, M. Wieser, Lokamani, J. Wolf, M. Helm, S. Gemming, J. Kerbusch, E. Scheer, T. Huhn, A. Erbe, Adv. Sci. 2015, 2, 201500017.

[12] S. J. van der Molen, R. Naaman, E. Scheer, J. B. Neaton, A. Nitzan, D. Natelson, N. J. Tao, H. van der Zant, M. Mayor, M. Ruben, M. Reed, M. Calame, Nat. Nanotechnol. 2013, 8, 385389.

[13] D. Dulic, S. J. van der Molen, T. Kudernac, H. T. Jonkman, J. J. D. de Jong, T. N. Bowden, J. van Esch, B. L. Feringa, B. J. van Wees, Phys. Rev. Lett. 2003, 91, 207402.

[14] N. Katsonis, T. Kudernac, M. Walko, S. J. van der Molen, B. J. van Wees, B. L. Feringa, Adv. Mat. 2006, 18, 1397-1400.

[15] S. J. van der Molen, H. van der Vegte, T. Kudernac, I. Amin, B. L. Feringa, B. J. van Wees, Nanotechnol. 2006, 17, 310-314.

[16] S. V. Snegir, A. A. Marchenko, P. Yu, F. Maurel, O. L. Kapitanchuk, S. Mazerat, M. Lepeltier, A. Leaustic, E. Lacaze, J. Phys. Chem. Lett. 2011, 2, 2433-2436.

[17] Arramel, T. C. Pijper, T. Kudernac, N. Katsonis, M. van der Maas, B. L. Feringa, B. J. van Wees, J. Appl. Phys. 2012, 111, 083716.

[18] Arramel, T. C. Pijper, T. Kudernac, N. Katsonis, M. van der Maas, B. L. Feringa, B. J. van Wees, Nanoscale 2013, 5, 9277-9282.

[19] G. Reecht, C. Lotze, D. Sysoiev, T. Huhn, K. J. Franke, ACS Nano 2016, 10, 10555-10562.

[20] R. Hania, A. Pugzל̧lys, T. Kudernac, H. Jonkman, K. Duppen, in Ultrafast Phenomena XIV: Proceedings of the 14th International Conference, Niigata, Japan, July 25-30, 2004 (Eds.: T. Kobayashi, T. Okada, T. Kobayashi, K. A. Nelson, S. De Silvestri), Springer Berlin Heidelberg, Berlin, Heidelberg, 2005, pp. 679-681.

[21] T. Kudernac, S. J. van der Molen, B. J. van Wees, B. L. Feringa, Chem. Commun. 2006, $3597-$ 3599. 
[22] A. J. Kronemeijer, H. B. Akkerman, T. Kudernac, B. J. van Wees, B. L. Feringa, P. W. M. Blom, B. de Boer, Adv. Mat. 2008, 20, 1467-1473.

[23] S. J. van der Molen, J. Liao, T. Kudernac, J. S. Agustsson, L. Bernard, M. Calame, B. J. van Wees, B. L. Feringa, C. Schoenenberger, Nano Lett. 2009, 9, 76-80.

[24] B. Wu, K. Ueno, Y. Yokota, K. Sun, H. Zeng, H. Misawa, J. Phys. Chem. Lett. 2012, 3, 14431447.

[25] A. Spangenberg, R. Metivier, R. Yasukuni, K. Shibata, A. Brosseau, J. Grand, J. Aubard, P. Yu, T. Asahi, K. Nakatani, Phys. Chem. Chem. Phys. 2013, 15, 9670-9678.

[26] A. Fihey, F. Maurel, A. Perrier, J. Phys. Chem. C 2015, 119, 9995-10006.

[27] H. Nishi, T. Asahi, S. Kobatake, Phys. Chem. Chem. Phys. 2012, 14, 4898-4905.

[28] R. Klajn, J. F. Stoddart, B. A. Grzybowski, Chem. Soc. Rev. 2010, 39, 2203-2237.

[29] M.-M. Russew, S. Hecht, Adv. Mat. 2010, 22, 3348-3360.

[30] T. C. Pijper, T. Kudernac, W. R. Browne, B. L. Feringa, J. Phys. Chem. C 2013, 117, 1762317632.

[31] R. Takahashi, S. Kaneko, S. Fujii, M. Kiguchi, Adv. Nat. Sci.: Nanosci. Nanotechnol. 2015, $6,015006$.

[32] S. V. Snegir, P. Yu, F. Maurel, O. L. Kapitanchuk, A. A. Marchenko, E. Lacaze, Langmuir 2014, 30, 13556-13563.

[33] D. Sysoiev, A. Fedoseev, Y. Kim, T. E. Exner, J. Boneberg, T. Huhn, P. Leiderer, E. Scheer, U. Groth, U. E. Steiner, Chem. - Eur. J. 2011, 6663 - 6672.

[34] L. A. Zotti, T. Kirchner, J. C. Cuevas, F. Pauly, T. Huhn, E. Scheer, A. Erbe, Small 2010, 6, $1529-1535$.

[35] Y. Kim, T. J. Hellmuth, D. Sysoiev, F. Pauly, T. Pietsch, J. Wolf, A. Erbe, T. Huhn, U. Groth, U. E. Steiner, E. Scheer, Nano Lett. 2012, 12, 3736-3742.

[36] A. A. Khodko, V. V. Khomenko, O. D. Mamuta, I. P. Mukha, D. O. Sysoiev, T. Huhn, S. V. Snegir, N. M. Kachalova, Mol. Cryst. Liq. Cryst. 2016, 639, 64-70. 
[37] A. Khodko, V. Khomenko, Y. Shynkarenko, O. Mamuta, O. Kapitanchuk, D. Sysoiev, N. Kachalova, T. Huhn, S. Snegir, Chem. Phys. Lett. 2017, 669, 156-160.

[38] D. Kim, H. Jeong, W.-T. Hwang, Y. Jang, D. Sysoiev, E. Scheer, T. Huhn, M. Min, H. Lee, T. Lee, Adv. Funct. Mater. 2015, 25, 5918-5923.

[39] Gold Nanoparticles for Physics, Chemistry and Biology (Eds.: C. Louis, O. Pluchery), Imperial College Press, London, 2012.

[40] B. V. Enustun, J. Turkevich, J. Am. Chem. Soc. 1963, 85, 3317-3328.

[41] J. Turkevich, P. C. Stevenson, J. Hillier, Discuss. Faraday Soc. 1951, 55-75.

[42] G. Frens, Nature (London), Phy. Sci. 1973, 241, 20-22.

[43] J. Kimling, M. Maier, B. Okenve, V. Kotaidis, H. Ballot, A. Plech, J. Phys. Chem. B 2006, $110,15700-15707$.

[44] During the immersion of the APTES coated cover slips the solution of AuNP should keep its color. If a color change occurs, the removal of physisorbed APTES was not successful. The whole process has to be repeated with fresh reagents.

[45] E. Pontecorvo, C. Ferrante, C. G. Elles, T. Scopigno, J. Phys. Chem. B 2014, 118, 6915-6921.

[46] Y. Ishibashi, T. Umesato, M. Fujiwara, K. Une, Y. Yoneda, H. Sotome, T. Katayama, S. Kobatake, T. Asahi, M. Irie, H. Miyasaka, J. Phys. Chem. C 2016, 120, 1170-1177.

[47] L. Guo, J. A. Jackman, H.-H. Yang, P. Chen, N.-J. Cho, D.-H. Kim, Nano Today 2015, 10, 213-239.

[48] R. E. Messersmith, G. J. Nusz, S. M. Reed, J. Phys. Chem. C 2013, 117, 26725-26733.

[49] P. K. Jain, K. S. Lee, I. H. El-Sayed, M. A. El-Sayed, J. Phys. Chem. B 2006, 110, 7238-7248.

[50] S. Snegir, I. Mukha, D. Sysoiev, E. Lacaze, T. Huhn, O. Pluchery, Materialwiss. Werkstofftech. 2016, 47, 229-236.

[51] P. Chen, B. Liedberg, Anal. Chem. (Washington, DC, U. S.) 2014, 86, 7399-7405.

[52] M. Homberger, U. Simon, Phil. Trans. R. Soc. A 2010, 368, 1405-1453. 
[53] O. Pluchery, E. Lacaze, M. Simion, M. Miu, A. Bragaru, A. Radoi, Optical characterization of supported gold nanoparticles for plasmonic biosensors. In Semiconductor Conference (CAS), 2010 International, 2010; Vol. 01, pp 159-162.

[54] H. Nishi, T. Asahi, S. Kobatake, J. Phys. Chem. C 2009, 113, 17359-17366.

[55] A. Fihey, F. Maurel, A. Perrier, Phys. Chem. Chem. Phys. 2014, 16, 26240-26251.

[56] A. Fihey, B. Kloss, A. Perrier, F. Maurel, J. Phys. Chem. A 2014, 118, 4695-4706.

[57] Z. Sekkat, in Photoreactive Organic Thin Films, Academic Press, San Diego, 2002, pp. 63104.

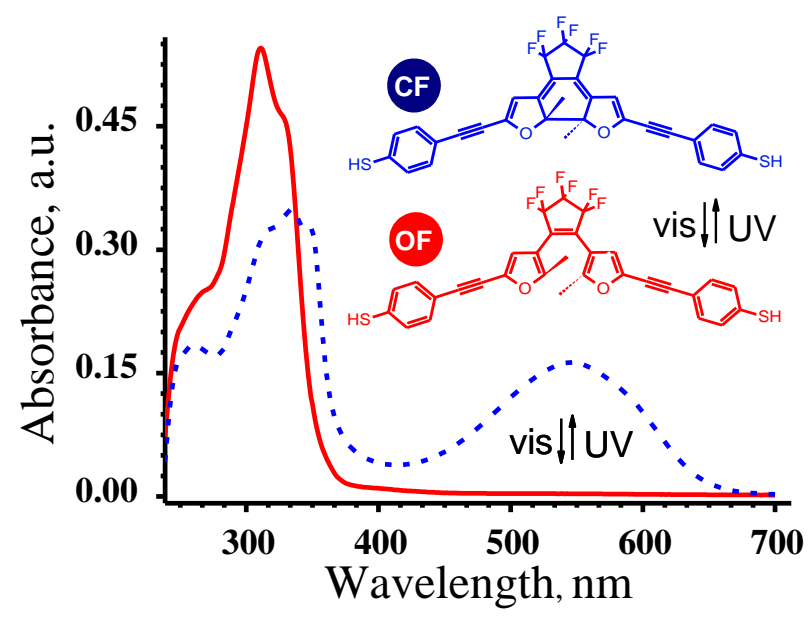

Figure 1. Temporal evolution of absorption spectra of $\mathrm{YnPhT}$ molecules in ethanol showing the reversible transition between $\mathbf{O F} \leftrightarrow \mathbf{C F}$ upon irradiation with either visible light or UV irradiation with $\lambda=254 \mathrm{~nm}$. Inset show structural changes in hexaflurocyclopentene photochromic core of YnPhT during ultraviolet/visible (UV/vis) light irradiation. 

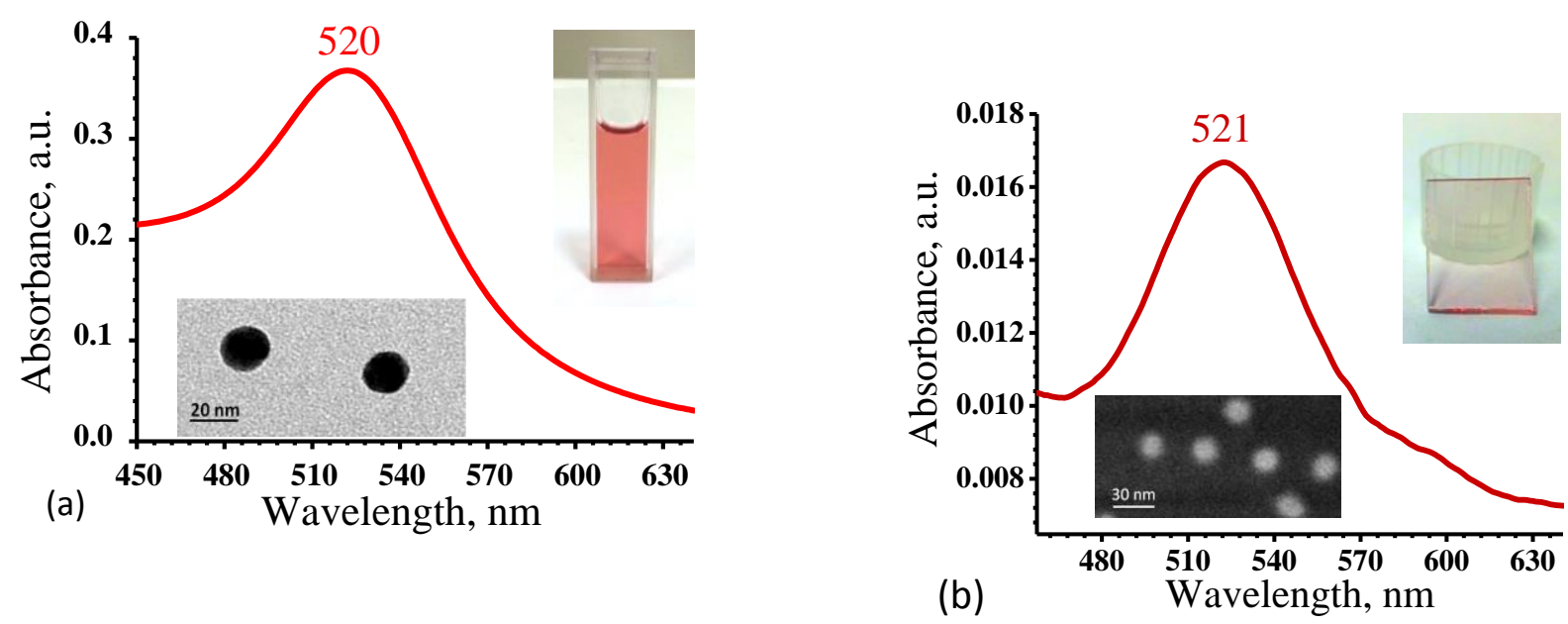

Figure 2. Absorption spectra of gold nanoparticles in (a) aqueous solution and on the (b) surface of a glass slide. Upper right inserts show pink color of (a) corresponding solution of AuNP in water and (b) on glass slide. Bottom inserts show (a) TEM image of AuNP physisorbed on a carbon coated copper grid and (b) SEM image of AuNP grafted via APTES on a glass slide.

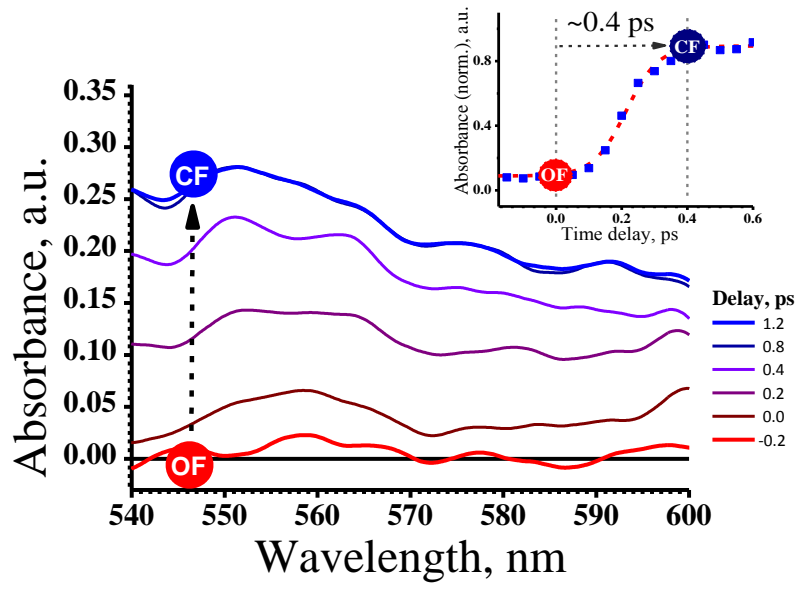

Figure 3. Time-resolved spectra of YnPhT molecules in ethanol solution displaying optical density changes during irradiation with $\lambda=266 \mathrm{~nm}$. Spectra marked $\mathbf{O F}$ and $\mathbf{C F}$ are spectra of molecules in $\mathbf{O F}$ before and spectra of molecules in CF after UV illumination. The inset is the normalized time profile of ring closing reaction extracted from time-resolved spectra at $\lambda=550 \mathrm{~nm}$ and fitted by deconvolution of exponential function and instrument response function (dotted line). 

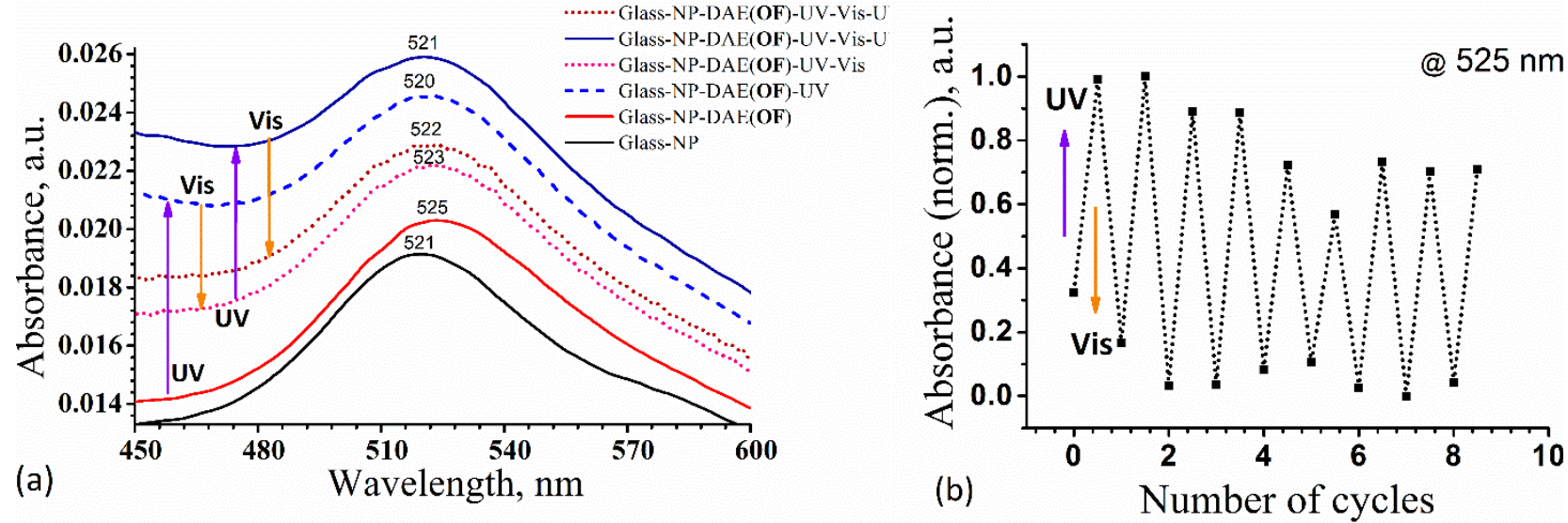

Figure 4. Absorption spectra of AuNP attached to glass slides. (a) Black curve (lowest) corresponds to bare AuNP on glass $\left(\lambda_{\max }=521 \mathrm{~nm}\right)$; red solid curve corresponds to AuNP with initially attached $\mathbf{O F}$ molecules $\left(\lambda_{\max }=525 \mathrm{~nm}\right)$ and solid blue curve corresponds to the same sample after two cycles of UV/visible irradiation $\left(\lambda_{\max }=521 \mathrm{~nm}\right)$. Duration of each irradiation is 6 min. Arrows define the stepwise variation of the absorption-spectra under consecutive UV/visible light irradiation. All spectra were measured on the same sample. (b) Profile of the light absorbance changes of glass-AuNP-YnPhT sample measured at $525 \mathrm{~nm}$ during consecutive UV/visible irradiation. UV irradiation leads to increased light absorbance whereas visible light irradiation leads to decreased absorbance.

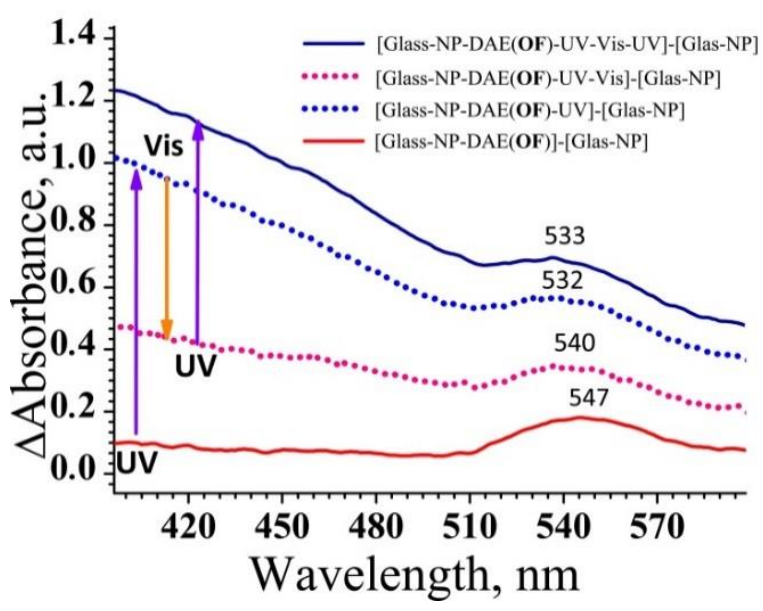


Figure 5. Difference spectra obtained by subtracting the signal of unmodified AuNP from the spectra of AuNP coated with YnPhT in the OF (red) and spectra obtained after two cycles of UV/visible light irradiation. 